BMC

Genomics

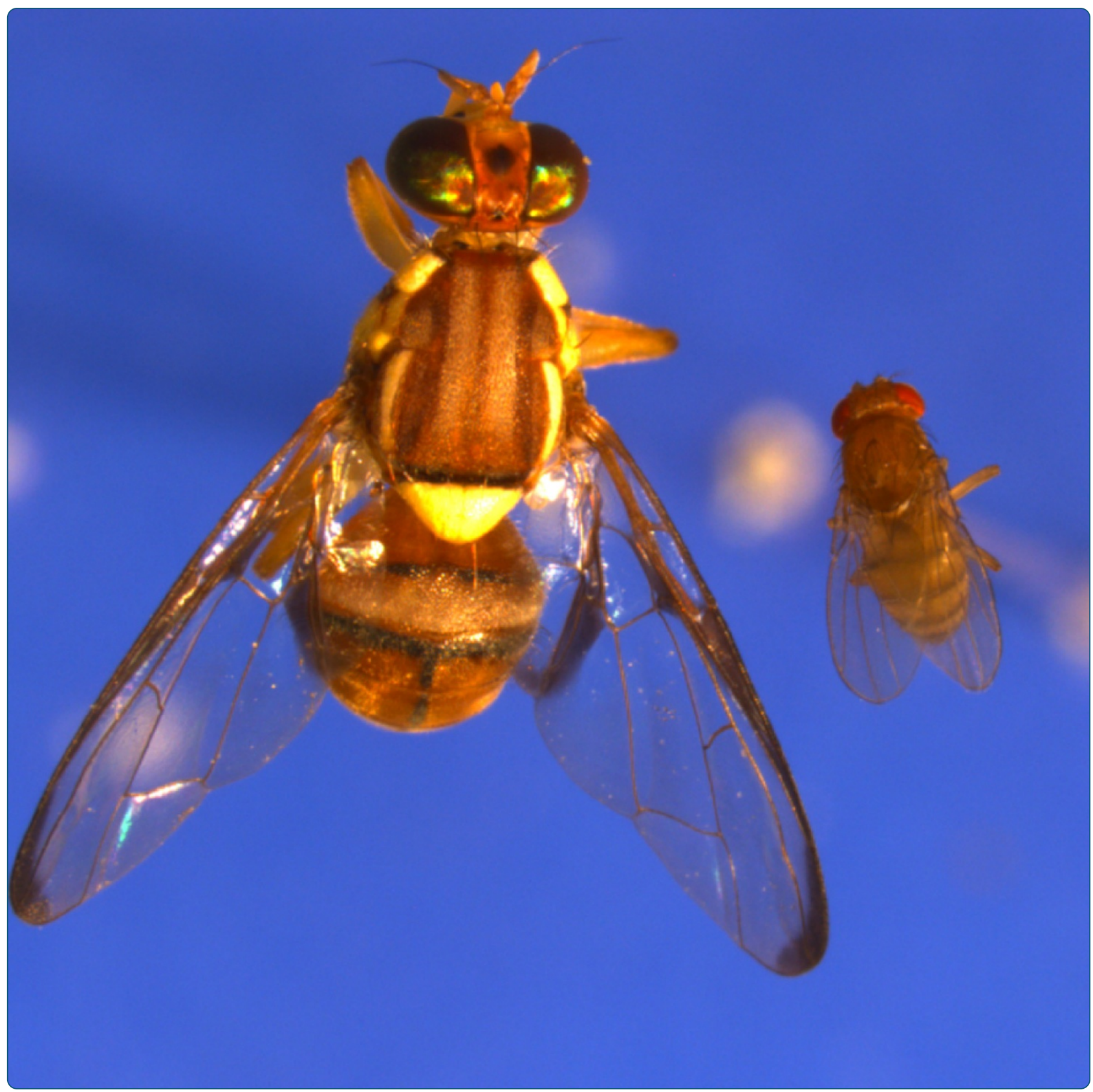

The draft genome of the pest tephritid fruit fly Bactrocera tryoni: resources for the genomic analysis of hybridising species

Gilchrist et al. 


\title{
The draft genome of the pest tephritid fruit fly Bactrocera tryoni: resources for the genomic analysis of hybridising species
}

\author{
Anthony Stuart Gilchrist ${ }^{1,2^{*}}$, Deborah CA Shearman ${ }^{1}$, Marianne Frommer ${ }^{1}$, Kathryn A Raphael ${ }^{1}$,
} Nandan P Deshpande ${ }^{3}$, Marc R Wilkins ${ }^{3,4,5}$, William B Sherwin ${ }^{1}$ and John A Sved ${ }^{1}$

\begin{abstract}
Background: The tephritid fruit flies include a number of economically important pests of horticulture, with a large accumulated body of research on their biology and control. Amongst the Tephritidae, the genus Bactrocera, containing over 400 species, presents various species groups of potential utility for genetic studies of speciation, behaviour or pest control. In Australia, there exists a triad of closely-related, sympatric Bactrocera species which do not mate in the wild but which, despite distinct morphologies and behaviours, can be force-mated in the laboratory to produce fertile hybrid offspring. To exploit the opportunities offered by genomics, such as the efficient identification of genetic loci central to pest behaviour and to the earliest stages of speciation, investigators require genomic resources for future investigations.
\end{abstract}

Results: We produced a draft de novo genome assembly of Australia's major tephritid pest species, Bactrocera tryoni. The male genome (650 -700 Mbp) includes approximately 150Mb of interspersed repetitive DNA sequences and 60Mb of satellite DNA. Assessment using conserved core eukaryotic sequences indicated $98 \%$ completeness. Over 16,000 MAKER-derived gene models showed a large degree of overlap with other Dipteran reference genomes. The sequence of the ribosomal RNA transcribed unit was also determined. Unscaffolded assemblies of $B$. neohumeralis and $B$. jarvisi were then produced; comparison with $B$. tryoni showed that the species are more closely related than any Drosophila species pair. The similarity of the genomes was exploited to identify 4924 potentially diagnostic indels between the species, all of which occur in non-coding regions.

Conclusions: This first draft B. tryoni genome resembles other dipteran genomes in terms of size and putative coding sequences. For all three species included in this study, we have identified a comprehensive set of non-redundant repetitive sequences, including the ribosomal RNA unit, and have quantified the major satellite DNA families. These genetic resources will facilitate the further investigations of genetic mechanisms responsible for the behavioural and morphological differences between these three species and other tephritids. We have also shown how whole genome sequence data can be used to generate simple diagnostic tests between very closely-related species where only one of the species is scaffolded.

\section{Background}

The discovery of the genetic processes causing and accompanying speciation has been a long-standing challenge for evolutionary biologists. However, good study systems are rare. Model study systems would ideally

\footnotetext{
* Correspondence: a.gilchrist@unsw.edu.au

${ }^{1}$ Evolution and Ecology Research Centre, School of Biological, Earth and Environmental Sciences, The University of New South Wales, Sydney, NSW 2052, Australia

${ }^{2}$ Sydney Grammar School, College Street, Darlinghurst, NSW 2010, Australia Full list of author information is available at the end of the article
}

include species that are firstly sympatric and secondly very recently diverged, with an effective mechanism of mating isolation. Additionally, the species should be amenable to forced mating in the lab to allow genetic analysis. In this paper, we present genomic resources to underpin the study of three sympatric tephritid fruit fly species that fulfil all these criteria. The three species are shown in Figure 1. For the major species, Queensland fruit fly, B. tryoni, we present a draft genome and annotation. For the two other sympatric species, B. neohumeralis and B. jarvisi, we present unscaffolded 


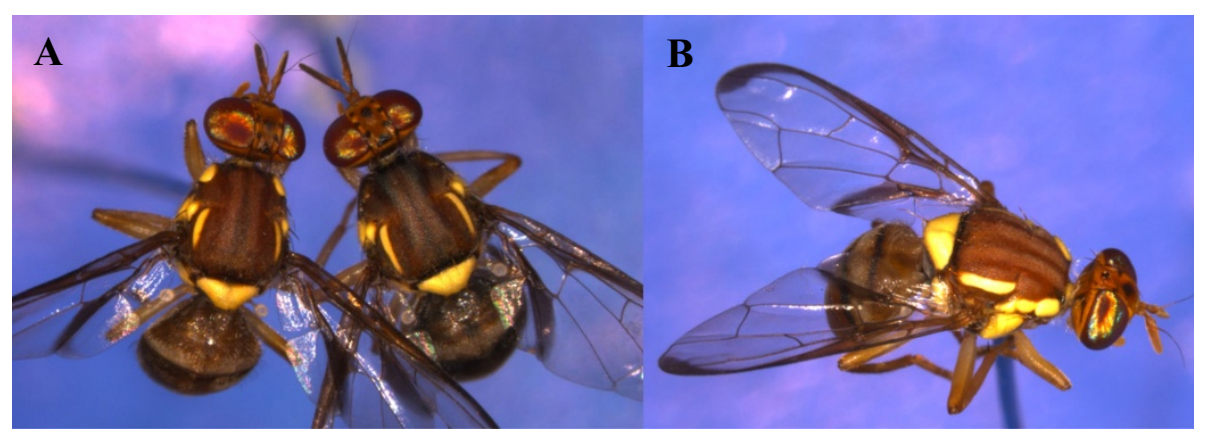

Figure 1 The Bactrocera species used in the present study. Panel A shows a B. tryoni male on the left and a male B. neohumeralis on the right. The species can be distinguished by the colour of the humeral calli (the "shoulder pads") on the anterior of the thorax, which is yellow in B. tryoni and dark in B. neohumeralis. B. neohumeralis usually have a darker body colour. Panel B shows a male B. jarvisi, which is distinguished from the other two species by the extra yellow marking immediately posterior to the humeral callus, the lighter body colour, clear costal cells on the anterior wing margin and abdominal stripes.

draft genomes to establish the genetic relations between the three species.

The economic importance of B. tryoni has prompted intense research interest for over 60 years. In Australia, B. tryoni is the most serious economic pest of horticulture and a major target for international and domestic quarantine efforts [1]. B. neohumeralis and B. jarvisi are also pests of economic importance and are sympatric with B. tryoni throughout most of tropical Australia [2]. Various Bactrocera species are major economic pests of fleshy fruit production in the Asia-Pacific region while ecologically comparable genera are major pests in other parts of the world: Ceratitis (including medfly) in Afrotropical regions, Anastrepha in Central and South America and Rhagoletis in Europe and North America [3].

$B$. tryoni and $B$. neohumeralis are identified by a morphological difference in the colour of the humeral calli (Figure 1) and a behavioural difference in time of mating: $B$. tryoni mates in a narrow window of falling light intensity at dusk, whereas $B$. neohumeralis mates in bright light during the middle of the day [4-6]. A DNA microsatellite-based survey found no evidence of hybridisation between the species in the wild [7]. However, interspecies hybrids can readily be produced in the laboratory by caging males of one species with females of the other (in both directions) [4]. These hybrids are viable and fertile, and can be maintained indefinitely. Not surprisingly, $B$. tryoni and B. neohumeralis appear extremely similar in DNA comparisons. Previous sequencing of nuclear and mitochondrial sequences found evidence of only one potential fixed difference in a ribosomal spacer region, ITS2, among a large range of shared polymorphisms $[8,9]$.

The third species is Jarvis' fruit fly, Bactrocera jarvisi, which is an emergent pest of a number of cultivated fruits in northern Australia. It has a more restricted range of host-fruits [2] and appears to be limited in geographic distribution by the distribution of its main endemic host, Planchonia careya [10]. B. jarvisi is morphologically quite distinct from B. tryoni and B. neohumeralis (Figure 1) and has been placed in a different subgenus of the Bactrocera [2]. DNA studies have shown that this different subgeneric status may not be warranted, but $B$. jarvisi is sufficiently differentiated that it has formed a convenient outgroup for DNA sequence comparisons between $B$. tryoni and $B$. neohumeralis [8]. Surprisingly, B. jarvisi can also be forced to mate with both $B$. tryoni and $B$. neohumeralis, with a substantial proportion of viable, fertile hybrids [11,12].

Therefore the three Australian pest tephritids, which can be hybridised and subjected to selection experiments, constitute a formidable model system that allows genetic and molecular analyses of a number of traits related to pest status - host fruit preferences, lure and odorant attractancy and invasive potential. Further, the apparent extreme similarity between $B$. tryoni and B. neohumeralis provides a model to investigate genome evolution and maintenance of separate species status, and the morphological differentiation of $B$. jarvisi allows investigation of the molecular mechanisms of morphological development and developmental canalisation.

\section{Results}

\section{Sequencing and de novo assembly}

The sequence data used for the de novo assembly of the male $B$. tryoni genome is summarized in Additional file 1 . The B. tryoni data consisted of $58 \mathrm{Gbp}$ of paired-end data, representing approximately 80 times coverage assuming a genome size of approximately $700 \mathrm{Mbp}$ (as calculated below). For the related species $B$. neohumeralis and $B$. jarvisi we obtained $62 \mathrm{Gbp}$ and $55 \mathrm{Gbp}$ of $100 \mathrm{bp}$ paired-end Illumina HiSeq data respectively. The assembly of the B. tryoni genome was performed using AbySS ver 1.3.4 [13] to construct contigs followed by scaffolding with SSPACE [14], as detailed in the Methods section. The statistics of the resulting assembly are shown in 
Table 1. Only five scaffolds containing bacterial sequences were detected and these were removed from the assembly. Due to a lack of mate pair data, B. neohumeralis and $B$. jarvisi remained unscaffolded.

To assess the completeness of the coding regions in the genome assembly, we used the CEGMA set of conserved orthologs [15]. Complete sequences were found for $93.6 \%$ of the 482 genes that constitute the core gene set. Inclusion of partial matches increased that percentage to $98.8 \%$, indicating that the assembly of coding regions was near-complete. Only $9.9 \%$ of matches had more than one ortholog within the assembly suggesting that the assembly contained only a low level of alternate coding assemblies. The mitochondrial genome of $B$. tryoni emerged from the assembly as a single scaffold with $99 \%$ identity with a complete $B$. tryoni mitochondrial genome [16], with our scaffold containing a possible 300 bp duplication in the $\mathrm{A}+\mathrm{T}$-rich region.

The same CEGMA-based approach was used to assess the completeness of the two unscaffolded assemblies. The B. neohumeralis assembly was $84.7 \%$ complete $(96.4 \%$ including partial matches). The $B$. jarvisi assembly was $87.1 \%$ complete (95.6\% including partial matches). However, for B. neohumeralis and B. jarvisi 23\% and $21 \%$ respectively of loci had more than one ortholog. That increase from $9.9 \%$ in B. tryoni suggested that there were more redundant contigs in both those assemblies.

\section{Genome size}

We first used the k-mer method to estimate genome size [17], using both Jellyfish [18] and DSK 1.6066 [19] to count 18-mers. For a male-only sample of B. tryoni, the calculated genome size was $797 \mathrm{Mbp}$. Recently, a sample of female-only DNA was sequenced (also Illumina $\mathrm{Hi}$-Seq) giving an estimate of $829 \mathrm{Mbp}$. The larger female genome size was expected on the basis of cytological evidence [20]. However, since these estimates were larger than those expected from other Bactrocera genomes [21,22], we sought alternate estimates of genome size.

Our alternative approach to the estimation of genome size was based on the coverage of putative transcripts,

Table 1 Statistics of the genome assemblies

\begin{tabular}{lccc}
\hline & B. tryoni & B. neohumeralis & B. jarvisi \\
\hline Number of scaffolds & 31957 & 287119 & 189607 \\
or contigs & & & \\
N50 length & 69525 & 2859 & 3879 \\
N50 number & 1590 & 72665 & 36685 \\
Longest & 3181581 & 85617 & 908794 \\
Total bp & 518958810 & 710077868 & 582862706
\end{tabular}

Note that only the $B$. tryoni genome was scaffolded. with the assumption that many transcripts originate from single copy sequences [23]. That assumption was supported in the case of the B. tryoni assembly by the low percentage of orthologs (9.9\%) in the CEGMA gene set. We first estimated coverage of all 16710 B. tryoni MAKER-derived transcripts (see below). We refined this measure to minimise the influence of repetitive sequences and erroneous gene models. Using a subset of 3310 filtered transcripts, we obtained a distinct peak coverage at 42.5 (Figure 2). Variation in stringency of mapping resulted in only a small variation in coverage estimates in the range 41-43. Given that the Illumina HiSeq data contained 29.8 GB of sequence, the estimated genome size was $701 \mathrm{Mbp}$. This was larger than previous estimates for other Bactrocera species of 445 and 619 Mbp, but similar to that of another tephritid Ragoletis juglandis [21]. However, if the 9.9\% of the CEGMA genes that had more than one ortholog were misassemblies rather than true orthologs, then the method may overestimate genome size by as much as $10 \%$.

\section{Repetitive sequence analysis}

Analyses of repeated sequences in de novo eukaryotic assemblies are sometimes limited to standard implementations of either homology-based searches (e.g. RepeatMasker) or de novo prediction (e.g. RepeatModeler [24]). However, we know that the genomes of B. tryoni, B. neohumeralis and $B$. jarvisi contain abundant insertions of mariner elements [25], and that partial sequences of other repeated elements have been found within sequenced introns (for example [26]). Also, satellite DNA sequences receive little attention despite their potential importance to many

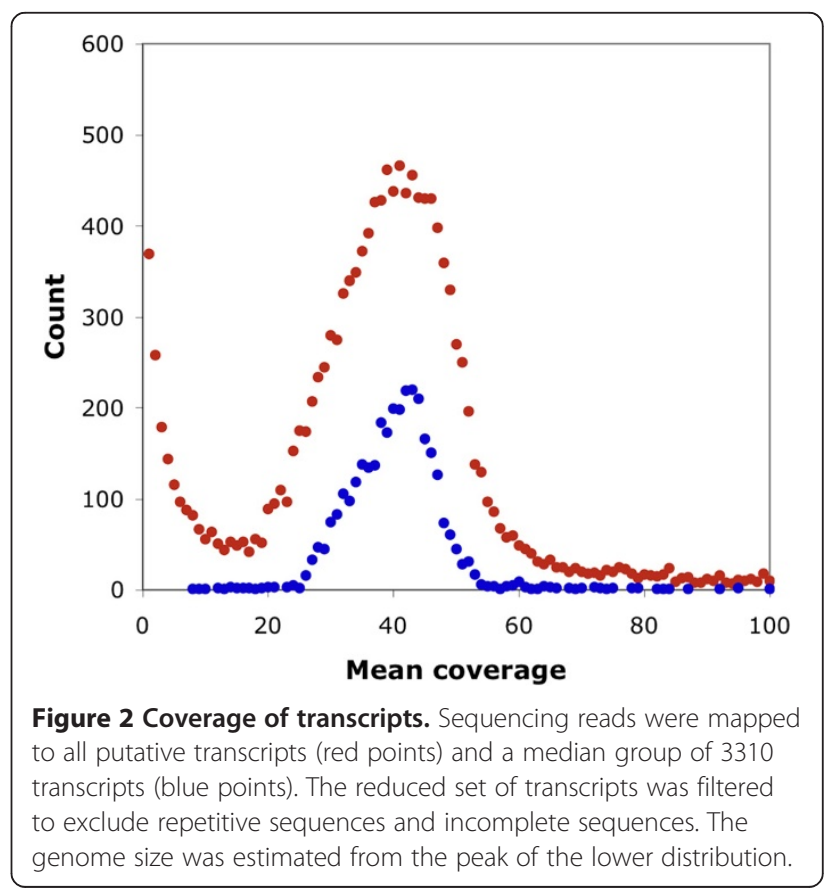


aspects of genome evolution and regulation [27]. Therefore, we undertook a detailed analysis of the tandem and dispersed repetitive sequences in B. tryoni.

We identified $B$. tryoni repetitive sequences from a combination of RepeatModeler de novo predictions, a kmer extension analysis and manual curation as detailed in the Methods section. Initially, RepeatModeler produced 1236 sequences totalling 1.8 Mbp. The k-mer extension analysis was similar to the approach used in [28], in which the highest frequency k-mers found in the raw reads were extended one base pair at a time using the highest abundance k-mers that overlapped the original $\mathrm{k}$-mer by $(\mathrm{k}-1)$ bases. The use of raw reads greatly increases the likelihood of finding repeats, such as satellite DNA, that are not easily incorporated into an assembly. Our k-mer extension produced hundreds of fragments in the range $50-300 \mathrm{bp}$. Among the 15 most abundant sequences were five potential satellite DNA sequences. In $B$. tryoni, these sequences ranged from $154 \mathrm{bp}$ to $182 \mathrm{bp}$, typical of alphoid satellite DNA [27]. For both $B$. neohumeralis and B. jarvisi, the k-mer extension analysis identified variants of the same five satellite sequences (Table 2). The k-mer extension approach also refined the RepeatModeler output, which identified the same satellite sequences but in a variety of tandem arrays of 2-5 repeats. The remaining sequences produced by the k-mer analysis were mostly fragments of transposons. Table 3 summarises the classification and abundance of those elements.

The genomic arrangement of the putative satellite sequences (tandem arrays, head-to-tail, head-to-head etc) was again investigated in the raw reads. The analysis described in the Methods section showed that these putative satellite sequences were predominantly in large head-totail tandem arrays, typical of satellite DNA. Analysis of higher order patterns in the tandem arrays is an objective of future work.

The final step in the identification of B. tryoni-specific repeats was a manual curation using an iterative alignment and extension of the remaining RepeatModeler de novo sequences. That process identified the consensus of mainly transposon-related sequences. The final output was a set of $153 \mathrm{~B}$. tryoni-specific repeat sequences ranging between 52 and 7328 bp in length, with a total length of $249 \mathrm{~kb}$. These sequences were classified on the basis of homology to Dipteran Repbase sequences [29] and are presented in Additional file 2. To estimate proportion of the genome consisting of those 153 repetitive sequences, we mapped the 298 million B. tryoni Illumina HiSeq reads to the $B$. tryoni-specific repeats. This suggested that approximately one third of the $B$. tryoni genome consists of repetitive DNA ( $\sim 31.4 \%$ of reads mapped to the repeats with mapping quality $q>20, \mathrm{NM}=4.9$ where NM is the SAM flag indicating the number of mismatches).

The utility of the $B$. tryoni-specific repeat library can be illustrated by comparing the masking ability of RepeatMasker [30] with and without the B. tryonispecific repeat library. Using RepeatMasker with default parameters and the Repbase repeat library [29], $31 \mathrm{Mbp}$ of the B. tryoni assembly was masked. Inclusion of our B. tryoni-specific repeat library increased the masking almost 5-fold to $146 \mathrm{MBp}$. The masking does not increase to $30 \%$ of the assembly since most satellite sequences and many transposon fragments were likely to be under-represented in the assembly.

Since $B$. neohumeralis and B. jarvisi both show very low sequence divergence from $B$. tryoni $(<1 \%$; see section in substitution rates below), homologs of the $B$. tryoni repeat sequences were constructed for the other two species by simply selecting the related repeats and substituting the most common single nucleotide polymorphisms or small indels from the second species. For each species, raw reads were then mapped to the appropriate set of repeats to estimate coverage for the repeated sequences. For B. neohumeralis, $33 \%$ of Illumina HiSeq reads mapped to the $B$. neohumeralis repeats (average $N M=5.2$ ). For B. jarvisi, $37.8 \%$ of reads mapped to the B. jarvisi repeats (average $N M=4.9$ ). Thus for each of the three species, approximately one third of the raw sequencing reads mapped to the $249 \mathrm{~kb}$ of repetitive sequences listed in Additional file 2.

Table 2 Abundance of the five main classes of satellite DNA

\begin{tabular}{lccccc}
\hline & & B. tryoni & & B. neohumeralis & $\begin{array}{c}\text { B. jarvisi } \\
\text { Satellite }\end{array}$ \\
\cline { 2 - 4 } Canonical elements & Variable elements & Total Mbp & Variable elements & Variable elements \\
\hline Btry_Sat1 (166 bp) & 68350 & 250000 & 41.5 & 195000 & 225000 \\
Btry_Sat263 (182 bp) & 19682 & 50000 & 9.1 & 37500 & 111000 \\
Btry_Sat274 (177 bp) & 12974 & 47000 & 8.3 & 39800 & 9500 \\
Btry_Sat2169 (154 bp) & 3332 & 17500 & 2.7 & 13000 & 11000 \\
Btry_Sat2877 (174 bp) & 4374 & 5000 & 0.9 & 29000 & 30000 \\
\hline
\end{tabular}

The Variable Elements count refers the total numbers of elements that vary from the canonical sequence by a single nucleotide substitution, insertion or deletion. Total Mbp is the total length of sequence of that satellite per genome calculated by multiplying the length of the element by the number of variable elements. For $B$. neohumeralis and B. jarvisi only the number of variable elements is shown since the genome size was not estimated. 
Table 3 Summary of the $B$. tryoni dispersed repetitive sequences

\begin{tabular}{lcc}
\hline & No. of elements & Total Mbp \\
\hline LINEs & 104159 & 49.18 \\
LTR Retroviral & 19836 & 8.44 \\
DNA transposons & 153861 & 46.29 \\
Unclassified & 150385 & 41.08 \\
Low complexity and simple repeats & 7827 & 0.81 \\
\hline
\end{tabular}

The number of elements was estimated using RepeatMasker with the repeat library prepared in this study.

Ribosomal RNA (rRNA) genes are expected to occur in large tandem arrays containing hundreds of copies of the loci. Sequence heterogeneity among the many loci affects assembly from short genomic sequencing reads [31]. Consequently, we manually assembled the consensus rRNA transcribed unit using similar methods to those used to assemble the other classes of dispersed repetitive DNA, an approach previously used with Drosophila [31]. The Intergenic Spacer regions (IGS) joining the transcribed units were not completely assembled due their highly repetitive nature. The rRNA consensus sequences for each of the three Bactrocera species showed the usual tandem arrangement of $18 \mathrm{~S}, 5.8 \mathrm{~S}, 2 \mathrm{~S}$ and $28 \mathrm{~S}$ genes. Transcriptomic assemblies confirmed our assembled sequence of the B. tryoni transcribed unit (DCAS, unpublished data). The alignment of the three sequences, along with the D. melanogaster rRNA sequence, is included in Additional file 3, which confirms the difference between the B. tryoni/B. neohumeralis ITS2 found in an earlier study [8]. Figure 3 shows the occurrence of sequence variants for the three Bactrocera species (we use "variant" to indicate sequence differences both within and between individuals of each species). In all three species, variation was reduced in the $28 \mathrm{~S}$ and $18 \mathrm{~S}$ regions with a greater reduction in the $28 \mathrm{~S}$ locus. Our results parallel those for Drosophila [31], where higher frequency variants $(>5 \%)$ tended to be concentrated in the Internal Transcribed Spacer (ITS) and IGS regions.

Comparisons between the three Bactrocera species showed that $B$. tryoni and $B$. neohumeralis have relatively few inter-species rRNA sequence differences. $B$. jarvisi shows greater differentiation from both $B$. tryoni and B. neohumeralis, particularly in the ITS and IGS. The differentiation of the $B$. jarvisi IGS was sufficient to prevent meaningful sequence alignment with the IGS of the other two species.

The amount of sequence variation also differed between the three species (Figure 3). The incidence of variants in the $B$. tryoni sequence reads was almost twice that of B. neohumeralis. Variation in the B. jarvisi data was as low as $B$. neohumeralis in the transcribed rRNA unit but higher than B. tryoni in the IGS and external transcribed spacer (ETS) region. The extra variation in B. tryoni was unlikely to have resulted from differences in levels of inbreeding because pooled sequencing data from 12 wild caught individuals showed no increase in

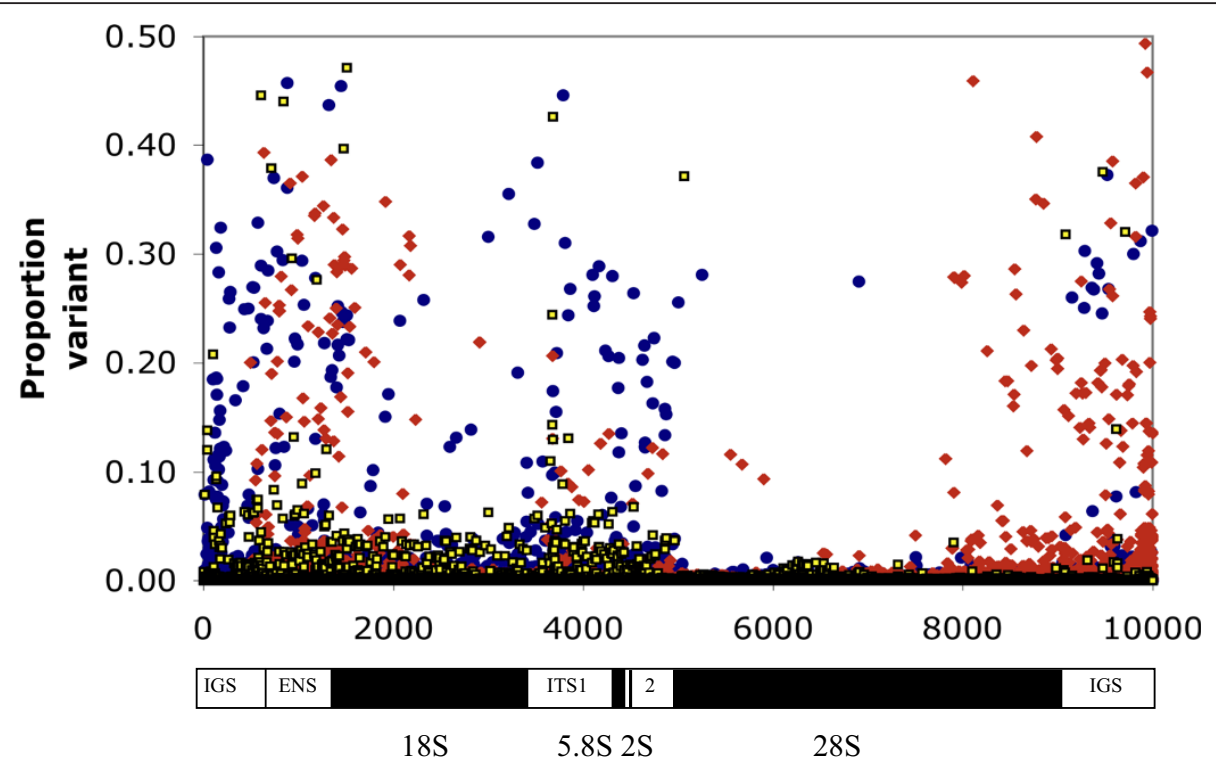

Figure 3 The comparative frequency of variants across the rRNA locus. The proportion of sites that vary from the consensus rRNA sequence is shown for B. tryoni (blue), B. neohumeralis (yellow) and B. jarvisi (red). Variation was measured from the consensus sequence for that species. The consensus sequence for B. jarvisi is only $9498 \mathrm{bp}$ (rather than $10 \mathrm{~kb}$ ) due to shorter assembled IGS sequences. The bar below the graph indicates the rRNA unit, with the four rRNA loci shown in black. The limits of the four rRNA loci are based on the D. melanogaster sequence. IGS=intergenic spacer (incomplete), ETS = external transcribed sequence, ITS1 and $2=$ internal transcribed sequences. 
heterogeneity over the sequencing data from our highly inbred strain of B. tryoni (data not shown), suggesting that the extra heterogeneity exists among the rRNA repeats of any one individual. The selective pressures operating on rRNA sequence variants both between and within individuals that may produce these varying patterns of heterozygosity are unclear.

\section{Annotation}

Annotation of the B. tryoni genome was performed using the MAKER pipeline [32]. Evidence use to create gene models comprised de novo transcriptomes and gene models from $C$. capitata and D. melanogaster. For repeat masking, we used a combination of the Repbase Dipteran library [29] and the B. tryoni-specific repeats identified above. Default parameters were used except that potential intron length was extended to $40 \mathrm{kbp}$.

The set of 16710 gene predictions produced by MAKER were subsequently classified using a local installation of Blast2Go [33]. Of the 16710 input sequences, 14334 produced Blast alignments with an NCBI invertebrate reference sequence library. 9417 of those gene models were successfully annotated with Gene Ontology terms. 11333 sequences were annotated with InterProScan. That same annotation process was repeated for the C. capitata and D. melanogaster gene models and the comparison of the resulting classifications is shown in Figure 4. The similarity of these classifications suggests that our assembly and annotation are reasonably complete. However, differences in the number of gene models for each species and the greater representation of Drosophilids in the databases used by InterProScan mean that at this stage it would be difficult to reliably interpret the relative differences in the Gene Ontology terms.

\section{Comparison with other Diptera}

To assess the overall composition of the B. tryoni gene model set, we compared it to gene model sets from two other Dipterans: C. capitata and D. melanogaster. Table 4 shows that the sizes (amino acid residues) of the 16710 $B$. tryoni gene models was comparable to C. capitata and D. melanogaster.

To comparatively assess the overall composition of our B. tryoni gene models, we used OrthoMCL [34], which groups transcripts by sequence similarity. Although the three sets of gene models contain different numbers of gene models (Table 4) reflecting the different annotation history and stage of curation, this comparison provides an indication of the overall completeness of our assembly. OrthoMCL produced 11688 orthologous groups and Figure 5 shows the overlap of those groups between the three species. Of the orthologous groups, 65\% contained representatives from all three species and only $3 \%$ of groups were B. tryoni-specific. While that high degree of overlap suggests that the B. tryoni assembly is reasonably complete, a caveat is that the C. capitata and $D$. melanogaster gene models were included in the B. tryoni MAKER annotation as part of the evidence used to create the $B$. tryoni gene predictions. Nevertheless, our B. tryoni gene models do not appear to be a simple subset of the other two species, since both $B$. tryoni and $C$. capitata are equally distinct from $D$. melanogaster and each other.

Within each ortholog group, closely-related species would be expected to have a similar numbers of gene models. As a result, the ratio of gene models within groups should be approximately 1:1. For each orthologous group, we calculated that ratio for each species pair. Figure 6 shows that, of the three pairwise comparisons, B. tryoni and C. capitata had the most groups with an approximate 1:1 ratio. Presumably that result is due not only to similarity of the species but also to the similar annotation methods used. Both pairwise comparisons involving $D$. melanogaster showed more groups with a $1: 2$ ratio rather than a $1: 1$ ratio. This must be due in part to the greater number of gene models available for $D$. melanogaster. Despite that bias, $80-84 \%$ of ratios were in the range $1: 3$ to $1: 1$, indicating that most ortholog groups contain similar number of gene models.

The possibility of gene enrichment was investigated in the 341 orthologous groups that contained only B. tryoni gene models. Those groups contained 1211 gene models (median sequence length $=150$ amino acids), which were annotated with Gene Ontology and Pfam terms using Blast2Go. Of the longer sequences, less than 50\% were either fragments of gene models in multi-species ortholog groups or transposon-related sequences. The possibility that the remaining gene models represent novel B. tryoni genes is a subject of future investigation.

\section{Substitution rates between Bactrocera species}

The small genetic distance between B. tryoni and B. neohumeralis was reflected in the nucleotide substitution rates between the two species. We partitioned fixed inter-species nucleotide substitutions between exons, introns, UTRs and non-coding DNA, with exon substitution rates being further classified as synonymous or nonsynonymous (Table 5). The observed substitution rates between B. tryoni and either B. neohumeralis or B. jarvisi were lower than that reported between, for example, Drosophila species (e.g. [35]). The non-synonymous versus synonymous ratio $\mathrm{d}_{\mathrm{N}} / \mathrm{d}_{\mathrm{S}}\left(\right.$ or $\left.\mathrm{K}_{\mathrm{A}} / \mathrm{K}_{\mathrm{S}}\right)$ was approximately 0.1 , which is consistent with some degree of purifying selection affecting each species.

In the raw B. neohumeralis Illumina HiSeq data, 197 instances of apparently novel stop codons were found (Table 5; Additional file 4). The majority of these occurred either in alternate transcripts (45) or sequences that had no 


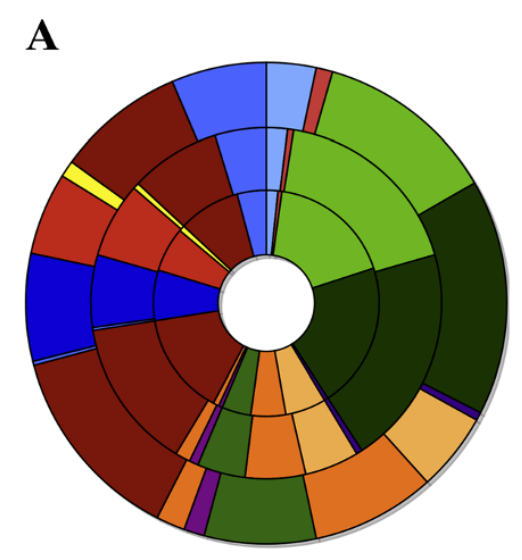

$\square$ reproduction

immune system process

metabolic process

- cellular process

- biological adhesion

$\square$ signaling

multicellular organismal process

developmental process

घrowth

locomotion

asingle-organism process

arhythmic process

response to stimulus

- localization

$\square$ multi-organism process

שbiological regulation

component organization/biogenesis

\section{B}

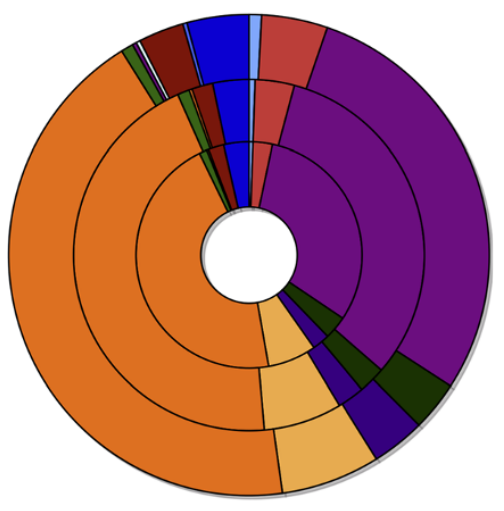

C

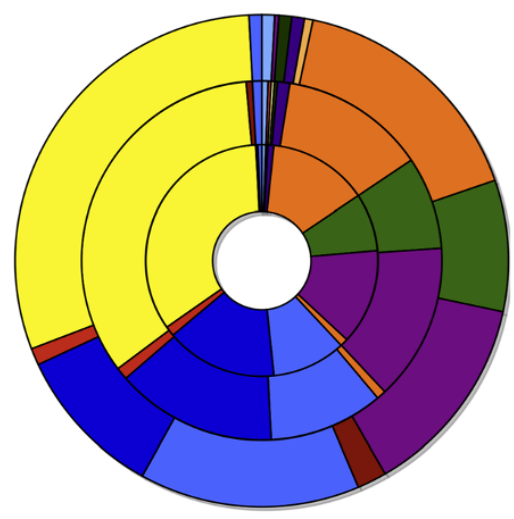

$\square$ protein binding transcription factor

nucleic acid binding transcription factor

- catalytic

- receptor

Estructural molecule

$\square$ transporter

abinding

Шelectron carrier

morphogen

antioxidant

Denzyme regulator

- translation regulator

molecular transducer

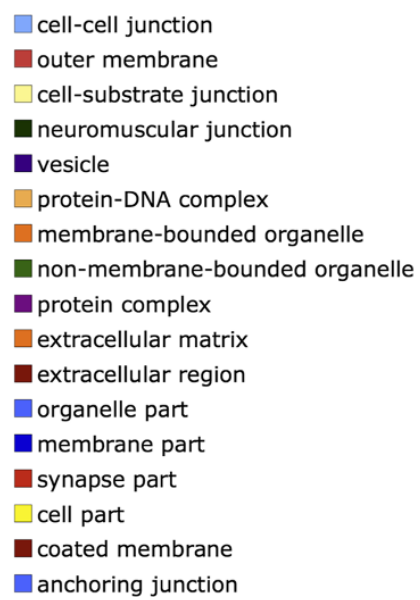

Figure 4 Gene Ontology comparison. Each graph compares the classification of the putative B. tryoni transcripts (outer ring) with the annotations of C. capitata (middle ring) and D. melanogaster (inner ring). Panel A: Biological processes. Panel B: Molecular Function. Panel C: Cellular components. The relative distributions support the conclusion that the assembly contains a near-complete assembly of coding DNA.

Blast results (70), while some stop codons were close to the end of the B. tryoni-version of the transcript. Differential expression of these is currently under investigation as part of a comparative transcriptomics project (K. Raphael pers. comm.). During investigation of these potential novel stop codons, we also found 30 instances where a substitution, if considered in isolation, would have produced a new stop codon. However, in each of those 30 potential transcripts, 
Table 4 Comparison of the number and size range of the gene models for the three Dipteran species shown

\begin{tabular}{lccc}
\hline & B. tryoni & C. capitata & D. melanogaster \\
\hline No. of gene models & 16710 & 20674 & 26950 \\
Mean length (aa) & 593 & 673 & 659 \\
Min. length (aa) & 21 & 21 & 11 \\
Max. length (aa) & 17244 & 20191 & 22949 \\
\hline
\end{tabular}

a second substitution in the same codon negated the effect of the first substitution.

The close similarity between the species at the sequence level allowed Illumina HiSeq data from both $B$. neohumeralis and $B$. jarvisi to be mapped against the B. tryoni assembly. For B. neohumeralis, $70.4 \%$ of reads mapped to the $B$. tryoni assembly with a mapping quality $q>20$. By comparison, only $41.8 \%$ B. jarvisi reads mapped to the B. tryoni assembly with quality $q>20$.

\section{Indel variation between Bactrocera species}

Since we did not have scaffolded assemblies for either B. neohumeralis or B. jarvisi, we were unable to reliably investigate syntenic differences between the species. However, the sequence similarity was exploited to identify a large number of deletions in the $B$. neohumeralis and $B$. jarvisi sequence data relative to the B. tryoni scaffolds. Gapped alignment programs (e.g bwa-mem) were used to identify B. tryoni scaffold segments with zero coverage. These were then filtered to extract only those

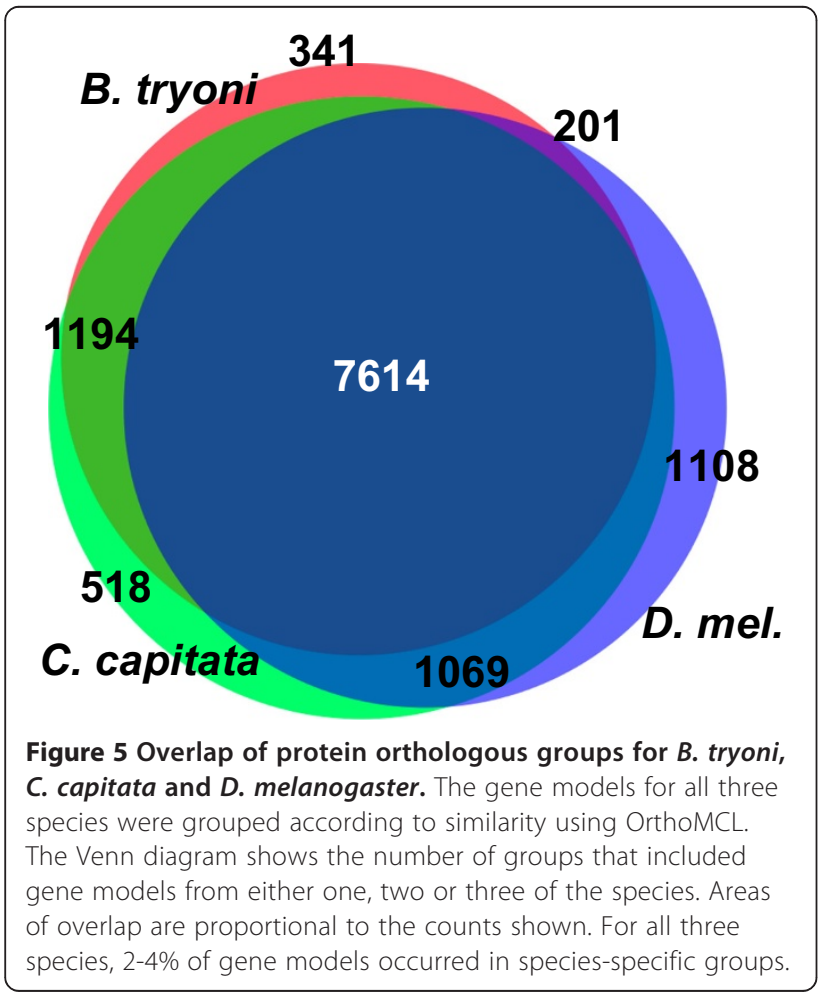

deletions with high, precisely aligned coverage on both sides of the deletion. For B. neohumeralis, of 59633 initial deletions over 10bp, only 4924 had sufficient coverage on both flanks to be investigated further. For B. jarvisi, 770662 initial deletions were reduced to 57285 .

Since transposons may play a significant role in sequence evolution, species as closely related as $B$. tryoni and $B$. neohumeralis provide an opportunity to investigate the possible involvement of transposons in the earliest stages of genome divergence. Using the deletions identified above, we extracted the 1000 bp genomic segments adjacent to each of the deletions identified in $B$. neohumeralis and B. jarvisi. A Blastn search of these flanking sequences, using the species-specific repeat library (requiring $80 \%$ identity over 80 bp length for a match), showed that $32 \%$ of sequences contained repetitive elements. However, this level of association between repetitive sequences and the deletions was not significant. Using the same Blastn parameters, 1000 sets of the same number of random genomic sequences (separated by the same distance) produced an average of $30.1 \%$ repeat hits. While that result coincided with our estimate of approximately $30 \%$ repetitive DNA in the B. tryoni genome, that figure was only coincidental since the repetitive DNA is not well represented in the assembly. Similarly, the same comparison between $B$. tryon $i$ and $B$. jarvisi also showed that repetitive DNA was not preferentially associated with deletions.

We also observed that many transposon sequences in the $B$. tryoni scaffolds were in homologous positions in both the $B$. neohumeralis and $B$. jarvisi contigs. We identified all mariner transposon sequences in the B. tryoni scaffolds, these were fragmented and often contained intervening non-transposon sequences. Any other mariner fragments within $1.5 \mathrm{~kb}$ (the average length of the mariner elements) were considered to be part of the same element. We then extracted the non-transposon sequences flanking each $B$. tryoni composite element and measured the length of the transposon insertion (i.e the distance between the two flanking segments). The distance between the homologous flanking sequences was then measured in B. neohumeralis or B. jarvisi (where the flanking sequences were on the same contig). This approach is illustrated in Figure 7. To assess the significance of any between-species differences, we extracted an equal number of random, paired $B$. tryoni sequences, with the insert sizes matching the gaps in the actual B. tryoni flanking sequence pairs. The results of this are shown in Figure $8 \mathrm{~A}$ and $8 \mathrm{~B}$. For both species pairs, the mean insert size was larger for the mariner sequences than control sequences $(B$. tryoni /B. neohumeralis 17 bp vs. 9 bp: 2 -tailed t-test, $\mathrm{p}<0.001$; B. tryoni /B. jarvisi $98 \mathrm{bp}$ vs. 29 bp: 2 -tailed t-test, $\mathrm{p}<0.001$ ) and the variances were correspondingly higher. This suggests that mariner sequence insertions are 


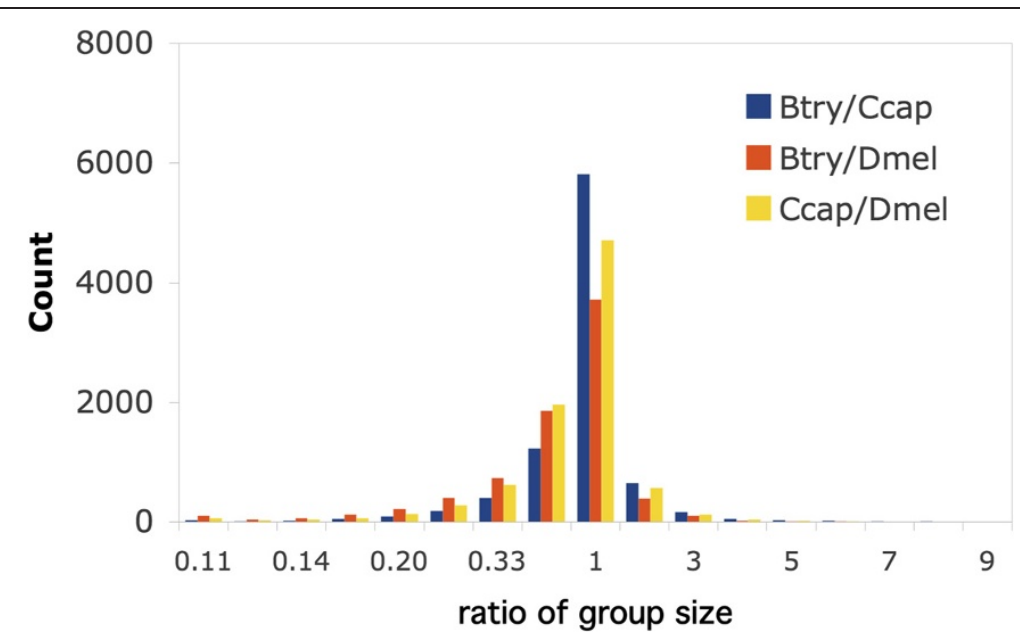

Figure 6 Relative size of orthologous groups of B. tryoni, C. capitata and D. melanogaster. The gene models within each orthologous group produced by OrthoMCL were classified according to species of origin. For each species pair, the ratio of the number of gene models was then calculated on a pairwise basis. The histogram shows the counts for the ratio indicated on the X-axis. For each species pair, the most common ratio was 1:1 indicating a strong correspondence of gene models between species. However, the ratios for $D$. melanogaster in particular are biased downward due to the greater number of gene models available for that species.

associated with a small increase of intra-species sequence variation. Similar comparisons for other repetitive elements are currently underway.

\section{Discussion}

The family Tephritidae consists of over 4000 species in over 400 genera [36], including major global economic pests [37]. Many of these species, including the Bactrocera, have arisen relatively recently in evolutionary terms [21]. Tephritids provide numerous promising study systems for speciation, behaviour, invasiveness and sex determination [38-40]. The present study presents a first set of Bactrocera-specific resources that will assist genomic and genetic studies in all these areas. In addition to the first draft of an annotated assembly of the B. tryoni genome, we have produced an extensive non-redundant library of B. tryoni repetitive DNA. Such a library not

Table 5 Substitution rates in $B$. neohumeralis and $B$. jarvisi in comparison to the $B$. tryoni assembly

\begin{tabular}{lcc}
\hline Region & B. neohumeralis & B. jarvisi \\
\hline Exon & 0.0032 & 0.0197 \\
Intron & 0.0061 & 0.0295 \\
$5^{\prime}$ & 0.0036 & 0.0259 \\
$3^{\prime}$ & 0.0026 & 0.0221 \\
Non-coding & 0.0072 & 0.0233 \\
Non-synonymous substitutions & 0.0012 & 0.0065 \\
Synonymous substitutions & 0.0021 & 0.0121 \\
Novel stop codons & 190 & 1329 \\
\hline
\end{tabular}

only assists annotation but also provides avenues for investigation of genome evolution. We have also delineated the majority of satellite sequences and have provided a complete assembly of the rRNA repeat unit, both of which are often lacking from de novo genome assemblies.

The close genetic similarity of B. tryoni and B. neohumeralis has attracted the attention of evolutionary biologists since the 1960s. This study quantified that similarity over the majority of the genome and confirmed previous first-generation sequencing studies of specific nuclear and mitochondrial regions $[8,9,41]$. The only differences identified in those earlier studies were two single-nucleotide substitutions and a trinucleotide indel within the ribosomal

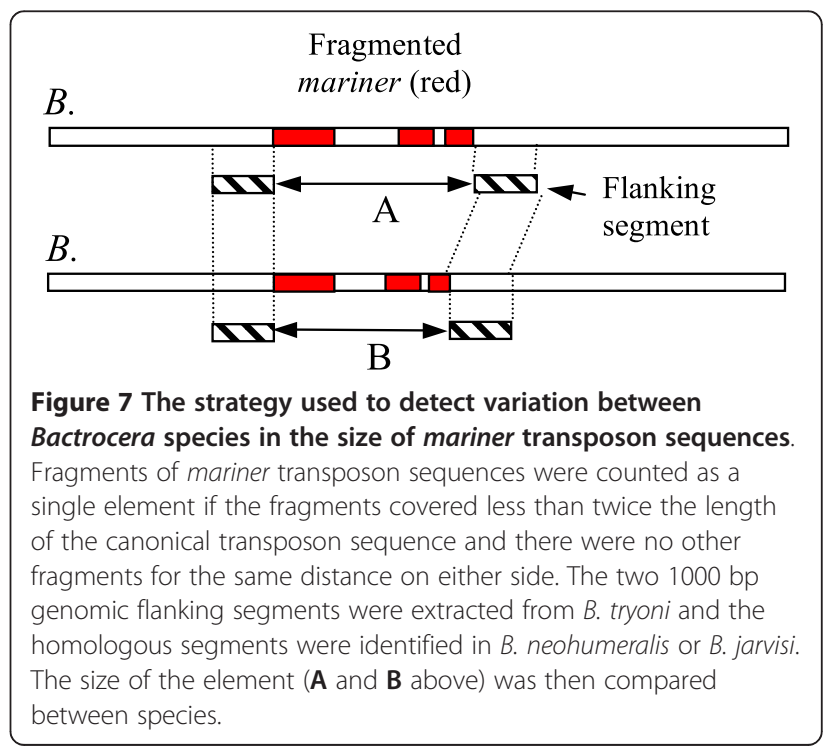




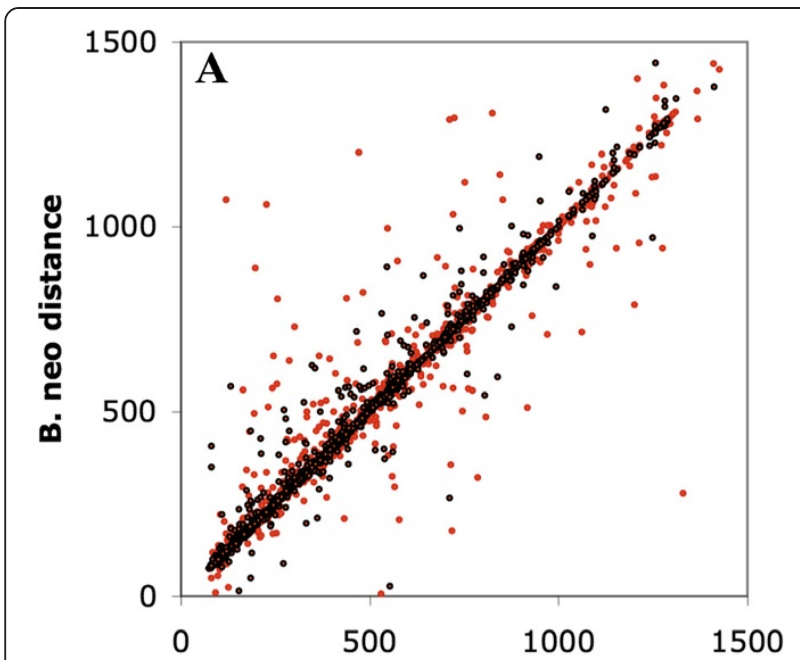

B. tryoni distance

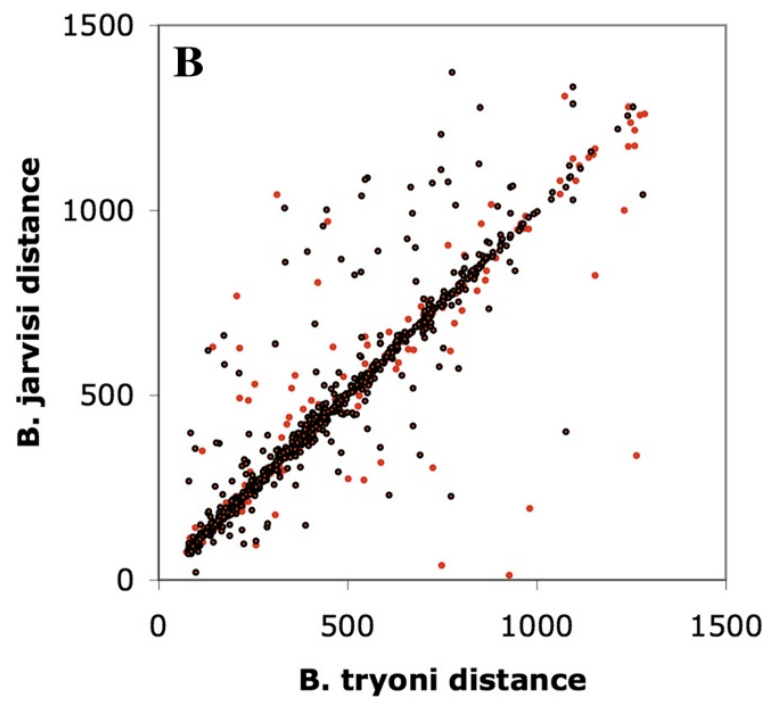

Figure 8 The comparison of the overall size of homologous transposon. Insertion sites in B. tryoni /B. neohumeralis (A) and B. tryoni /B. jarvisi (B). For each species pair comparison, homologous DNA segment size varies more in the vicinity of transposon (mariner) sequences (red points) than at random sites (black points). The control group consisted of an equivalent number of sequence fragments drawn at random from the two relevant assemblies.

gene internal transcribed spacer (ITS2), a level of differentiation more commonly observed between populations than between reproductively isolated species. Those differences were confirmed in the present study.

Our results have highlighted the potential for speciation studies in the Bactrocera genus. The sequence difference between $B$. tryoni and $B$. neohumeralis is considerably less than that found between species of Drosophila [35]. The closest species pairs are D. pseudoobscura - D. persimilis, a cross which produces sterile males [42] and D. simulans D. mauritiana. The estimated synonymous substitution rates for these two pairs are around 1.5\% [43] and 4.7\% [44], respectively, compared to our estimate of $0.21 \%$ for $B$. tryoni and $B$. neohumeralis. The estimate for B. tryoni and $B$. neohumeralis is sufficiently low as to be comparable with the extent of variation between strains of $D$. melanogaster. It is, however, difficult to measure genome-wide polymorphism levels with the currently available data. The B. tryoni strain used for sequencing was established from a mutant individual [45] and subsequently subjected to two further rounds of single-pair inbreeding to reduce polymorphism and facilitate assembly. The B. neohumeralis strain was not systematically inbred, but its establishment and maintenance as a laboratory strain for several years may have contributed to a loss of variability. Questions about the extent of polymorphism within and between the two species can only properly be answered by sequencing unrelated individuals from wild populations.

The close similarity between B. tryoni and B. neohumeralis is consistent with the fact that, despite the complete absence of wild hybrids [7], hybrids between the two species are viable and fertile, with only marginal reductions in fitness [4]. Somewhat surprisingly, laboratory hybrids between $B$. jarvisi and $B$. tryoni are also viable and fertile, despite the much greater genetic distance between the two (yet still comparable to the $D$. pseudoobscura - D. persimilis pair). In this case, the fertility of male hybrids is only slightly reduced ( $\sim 80 \%$ of non-hybrids) and the sex ratio of hybrid crosses in both directions is slightly biased. Crosses between $B$. jarvisi and B. tryoni may produce up to $70 \%$ females in some crosses (Gilchrist, unpublished observation). Viable and fertile hybrids can also be produced by forced mating between B. neohumeralis and B. jarvisi (Gilchrist and Belanto, unpublished observation). The potential use of hybrids, together with the genome information, provides a powerful system for investigating the genetic basis for all identifiable phenotypic differences between the three species, including mating-time differences and morphological divergence. The degree of genetic similarity between B. tryoni and B. neohumeralis is unprecedented amongst the closely-related dipteran species studied to date, thereby providing a model for investigating the earliest stages of speciation. This is a field in which good study systems are extremely rare [46-51].

We also point the way to overcoming the problem of diagnosis: in the past, the similarity between $B$. tryoni and $B$. neohumeralis prevented the development of diagnostic genetic markers for use in hybridisation studies and quarantine testing. Here, we present over 4000 small indels that, subject to studies in outbred wild populations, could provide the basis for new genetic markers. These deletions are currently being investigated as a basis for a simple PCR-based test to allow quarantine authorities to differentiate $B$. tryoni and B. neohumeralis (since there is currently no reliable test to split the two species at the larval stage). 
The methods we have used can be applied to any of the closely-related species groups which are challenging for traditional morphological taxonomy (e.g. the B. dorsalis species complex; [39]). Our method requires only a single assembled reference genome for comparison with raw sequencing reads from sibling species.

\section{Conclusions}

This paper presents a comprehensive first draft genome of an important horticultural pest, the Queensland fruit fly or Q-fly, B. tryoni. Although endemic to Australia, $B$. tryoni is an insect pest of worldwide concern, particularly in these times of burgeoning movement of people and produce. Further, it represents the first annotated and published genome of a species in the genus Bactrocera, which contains the great bulk of tephritid pests in Asia and Oceania. Our data provides an important basis for comparison with the genome of C. capitata and with other tephritid genome projects, for functions related to host-fruit recognition, invasive potential and developmental regulation. Our data will also have direct application to the delineation of recent radiations such as the $B$. dorsalis species complex found in Pacific and south-east Asian countries [39].

The $B$. tryoni genome has also enabled analysis of the genomes of two related Bactrocera species, B. neohumeralis and B. jarvisi. These are of interest because they are clearly distinct species with different behaviours leading to strong pre-mating isolation. Yet, since hybrids between these species are fertile, they present an unusually powerful model for investigating the genetic bases of morphological development, the evolution of morphological change and the molecular aspects of pest status and behaviour.

\section{Methods}

\section{Sample collection and DNA extraction}

The B. tryoni strain used for sequencing was the bent wings (bw) strain [45]. The $b w$ mutation was isolated from a single wild fly caught near Gosford, NSW, Australia, bred for homozygosity of the marker and maintained in the lab for 10 years (approximately 80 generations) before being further inbred by two rounds of single pair matings. The B. neohumeralis and B. jarvisi strains used for sequencing were caught near Cairns, Queensland, Australia in 2006 and maintained in lab culture since that time. Neither were deliberately inbred as was the B. tryoni strain. Genomic DNA for each sequencing run was extracted from 20 male fly heads using the method as described in [26].

\section{Genomic sequencing}

For Illumina HiSeq runs, libraries were prepared with a commercial kit (Paired-End DNA Sample Prep Kit; Illumina Inc., San Diego, CA, USA) following the manufacturer's protocol (Paired-End Library Construction). For B. tryoni, both Illumina paired-end sequencing (GAIIx, HiSeq and MiSeq) and 454 FLX Titanium pyrosequencing were performed at the Ramaciotti Centre at the University of NSW. For the mate-pair data, two Illumina GAII runs were performed at the Ramaciotti Centre at the University of NSW (3 kb insert), while 1 lane of Illumina HiSeq mate-pair data (10 kb insert) was generated at the Hawkesbury Institute for the Environment, University of Western Sydney. Additionally, a long jumping distance (LJD) mate-pair library (8 kb insert) was prepared by Eurofins MWG Operon, Ebersberg, Germany. The quality of paired-end data was assessed using FASTQ and subsequent quality trimming performed with the Trimmomatic software [52]. The Eurofins LJD library was quality trimmed by that company. Quake software [53] was used to remove and/or correct singleton 18-mers only in the Illumina HiSeq paired-end data (other data not having sufficient coverage).

For $B$. neohumeralis three lanes of $100 \mathrm{bp}$ paired-end Illumina HiSeq data were obtained, totalling $62 \mathrm{Gbp}$. Two of the libraries were prepared with $300 \mathrm{bp}$ insert size and one with $500 \mathrm{bp}$ insert size. For B. jarvisi, two lanes of 100 bp paired-end Illumina HiSeq data were used, one with $300 \mathrm{bp}$ inserts and the other with $500 \mathrm{bp}$ inserts. The maleonly data totalled $55 \mathrm{Gbp}$.

\section{Assembly}

After Quake-correction [53] of the Illumina HiSeq pariedend data, the B. tryoni contigs were generated from the paired-end GAII, paired-end Illumina HiSeq, paired-end Illumina MiSeq and Roche 454 FLX Titanium reads using the AbySS assembly program [ver $1.3 .4 ; 13$ ] with overlap length $k=65$. The $k$ value was determined after testing values ranging from 40-70. Contigs greater than $210 \mathrm{bp}$ were retained for scaffolding. Scaffolding was performed using the SSPACE scaffolder [14]. The three mate-pair libraries were added in order of increasing insert size $(3 \mathrm{~kb}$, $8 \mathrm{~kb}$ and $10 \mathrm{~kb}$ ) as advised by the SSPACE authors. We screened for the presence of bacterial sequences in our assembly using a Blastn search against the NCBI NT database (e-value 1e-06). When any of the top three hits were bacterial, the scaffold was removed. CEGMA was run according to the authors' instructions at http:// korflab.ucdavis.edu/datasets/cegma/. The B. tryoni scaffolds have been submitted to NCBI, with accession number JHQJ00000000.

\section{Genome size estimation}

We estimated genome size using two methods. First, we used the k-mer coverage methods (e.g. [17]), using both Jellyfish [18] and DSK [19] to count k-mers. Second, we used a variation of the approach of [23] to estimate genome size from the coverage of single copy sequences. 
The first step in that process was to map all Illumina HiSeq reads to the 16710 MAKER-derived transcripts using bwa-mem [54] and filtering out reads that mapped with low-quality $(q<30)$. The low coverage transcripts (coverage < 10) comprised 16\% of transcripts and were likely to include many truncated or erroneous transcript predictions. High coverage transcripts (coverage $>60$ ) comprised $14.6 \%$ of transcripts and were dominated by transcripts including highly repetitive sequences. The central peak of the distribution indicated a mean coverage of 41-43.

To refine this estimate, we re-analyzed the coverage of the transcripts for the median 50\% of transcripts ranked by coverage. Genomic scaffold segments corresponding to each transcript were extracted along with the flanking 100bp regions. Genomic segments were used for read mapping as they had no intron/exon boundaries and thus provided longer contiguous regions for mapping. Genomic segments containing runs of five or more Ns were removed, as were transcripts with a MAKERderived AED score $>0.2$ (indicative of transcripts with lesser evidential support). This resulted in a final set of 3310 genomic segments corresponding to individual putative transcripts. Raw reads were again aligned to the set of 3310 transcripts with a mapping quality filter of $q=55$ to ensure mappings were unique. Coverage was measured using BEDtools utility genomeCoverageBed [55], excluding the $100 \mathrm{bp}$ flanking regions. Repetitive sequences in introns resulted in small regions of very high coverage, 10-1000 times above the median. Consequently, the median coverage rather than the mean coverage was used. Variation of the mapping quality filter (e.g. $q$ as low as 10 or 20) resulted in only small changes in the coverage estimates.

\section{Repetitive sequence analysis}

RepeatModeler was run on the final B. tryoni assembly following the authors' instructions. The 18-mer extension method began with Jellyfish program [18], which was used to estimate 18-mer frequencies in the uncorrected Illumina HiSeq data. Using custom Perl scripts, we started with the most common 18-mer, which was extended by a single base pair after finding the next most common 18-mer that overlapped the starting 18-mer by $17 \mathrm{bp}$. This was repeated in both directions until an already-used 18-mer was met. No 18-mer was used more than once and only sequences that extended more than 50bp were retained for further analysis. The process was terminated after the 50000 most abundant 18-mers had been either been extended or eliminated due to inclusion in a previous extension sequence.

\section{Genomic arrangement of satellite sequences}

Since satellite sequences do not assemble easily, their arrangement in the genome (i.e. as tandem repeats or dispersed elements) was assessed in the $100 \mathrm{bp}$ Illumina reads rather than in the subsequent genome assembly. Although no single 100 bp read will cover a whole satellite monomer, if satellites are arranged in head-to-tail tandem arrays, then a predictable proportion of reads should cover the tail of one repeat unit followed by the head of the next repeat in the array. For example, two 12-mers at positions 1 and 50 in the satellite sequence should always appear 50 bp apart in the same 5'-to-3' orientation. Conversely, two 12-mers that are more than 88 bp apart, should rarely co-occur. Additionally, 12-mers near the 3' end of the satellite sequence should always occur in the negative direction (i.e. upstream) of the 1-position 12-mer. Other arrangements, such as headto-head or dispersed, will not show that same pattern. While we used 18-mers in other sections of this study, we used 12-mers to speed counting. Additional file 5 shows that $~ 92 \%$ of 12-mers from Btry_Sat1 were at the distance and in the orientation expected if Btry_Sat1 occurred mainly in large tandem arrays. Similar evidence was found that the other four satellite sequences were also present mainly as head-to-tail tandem arrays.

\section{Satellite sequence copy number}

To obtain a better estimate of satellite content of the $B$. tryoni genome, we estimated the frequency of satellite sub-sequences in the raw reads. We first counted the frequency of the 18-mers that comprise each of the six satellite sequences in the raw reads. Adjusting for coverage of the Illumina HiSeq reads, the mean frequency of all the non-overlapping 18-mers from each satellite gives an estimate of the number of genomic copies of the canonical sequence (Table 3). If searches allowed for variation by a single nucleotide substitution, more satellite sequences were detected. The data shown in Additional file 5, which includes single nucleotide substitutions, showed that the estimate of the number of Btry_Sat1 sequences (indicated by reads that had two distinct 12-mers at the correct spacing and in the correct orientation) increased from $\sim 70,000$ to over 250,000. Those variant sequences were also mainly contained within tandem arrays. For example, in Btry_Sat1, variants of the first and second 12-mers should co-occur on the same $100 \mathrm{bp}$ read at a frequency of 0.87 . This is the proportion of all the $100 \mathrm{bp}$ reads that contain 12-mer 1 and will also extend sufficiently in the 3' direction to include all of the adjacent 12-mer 2. For each million occurrences of Btry_Sat1 12-mer 1 in the Illumina HiSeq reads, we observed $\sim 700,000$ occurrences of 12-mer 2 on the same read.

\section{Dispersed repeats}

Dispersed repetitive sequences commonly consist of many fragments of canonical transposon sequences, with various 
degrees of sequence heterogeneity amongst the fragments. This inherent heterogeneity prevents good assemblies of those sequences. However, canonical versions of repeated sequences can be reconstructed directly from the sequencing reads e.g. the construction of Drosophila rRNA sequences [31]. To identify as many as possible of the underlying canonical sequences, we undertook a manual curation of the remaining RepeatModeler de novo sequences and the 18-mer extension sequences. That process began by performing a Blastn alignment of all the RepeatModeler de novo sequences against the B. tryoni scaffolds (80\% identity, e-value 1e-06). Starting with the fragment with the highest number of hits, we then performed an iterative process of alignment and consensus extension of the sequences. The process worked as follows. Each potential repeat sequence was aligned to the $B$. tryoni scaffolds using Blastn (80\% identity, e-value 1e-06). For each alignment, a custom Perl script extracted the matching scaffold (genomic) sequence along with up to $200 \mathrm{bp}$ of flanking sequence. Those extracted segments (often numbering several hundred) were aligned with Muscle [56] using the SeaView alignment viewer [57] allowing gapped alignments and $60 \%$ consensus threshold. The starting sequence could then be extended by up to $200 \mathrm{bp}$ depending on the length of the valid consensus sequence. The process could then be iterated until no further consensus extension was possible. Terminal inverted repeat sequences produced a common sequence linked to two (or sometimes more) consensus sequences. In those cases, each consensus was extended separately and pairs would eventually overlap if the original terminal inverted repeat sequences came from a similar repetitive element. The final extended sequence was then aligned with the remaining RepeatModeler de novo sequences (Blastn, $80 \%$ identity). This resulted in the culling of numerous related RepeatModeler de novo sequences, which in turn reduced the number of sequences that had to be examined. Eventually, the manual curation process was no longer feasible due to a combination of too few aligned genomic segments and increasing heterogeneity among those segments. Also, if taken too far, the 'align and extend' process could eventually start identifying conserved protein motifs as repetitive elements.

To finalise the consensus sequence, the most common variant at each SNP was checked by mapping the Illumina HiSeq reads to the candidate repeats using bwa-mem [54], extracting variant frequencies from the Samtools mpileup file [58] using VarScan 2 [59]. Sequences with greater than $80 \%$ identity to an existing repeat sequence were removed. Lastly, the percentage of raw reads mapping to those repeats with mapping quality $q>20$ was calculated from the alignment file. As a relative indicator of variation, the average number of mismatches for the mapped reads was calculated from the NM flag in the bwa-mem output.

\section{Species-specific repeat variants}

We first mapped reads from each species to the $B$. tryon $i$ repeats, retaining reads with mapping quality $q>20$. SNPs with $>50 \%$ frequency were extracted from the Samtools mpileup file [58] using VarScan 2 [59]. Those variants were then incorporated into the $B$. tryoni repeat sequences to produce a species-specific set of homologous repeats. Raw reads from each species were aligned to the appropriate set of repeats to estimate coverage for the repeats. Statistics were calculated only for reads with mapping quality $q>20$.

\section{Ribosomal RNA sequences}

The 'align and extend' process used to construct the canonical dispersed repeat sequences was also used to determine the sequence of the transcribed section of the rRNA repeat unit. The IGS sequence was incomplete and limited to the regions flanking the transcribed unit. The highly variable and repetitive nature of the IGS prevented the complete extension of the IGS sequence. All sequence variants for the transcribed rRNA unit and flanking IGS regions were extracted from the equivalent coverage of raw Illumina HiSeq data. Exact delineation of the rRNA genes was based on the D. melanogaster homologues [31].

\section{MAKER Annotation}

Within the MAKER pipeline, we used three gene predictors: SNAP [60], AUGUSTUS 2.5.5 [61] and GENEMARK [62]. SNAP was trained using the set of conserved genes identified by the CEGMA pipeline. The training for AUGUSTUS was generated using the whole $B$. tryoni genome, now available as a web-based service (http://bioinf. uni-greifswald.de/bioinf). GeneMark was self-trained. EST evidence was provided in the form of five B. tryoni de novo transcriptome libraries produced by the Trinity software [63]. Four of the libraries were assembled using separate RNA preparations from whole embryonic, larval, late pupal and adult individuals (D. Shearman unpublished). The fifth library was prepared from brain tissues. Protein homology evidence for MAKER consisted of coding sequences from two other Dipterans: D. melanogaster (ftp://ftp.flybase.net/ releases/FB2013_06/dmel_r5.54/fasta/dmel-all-CDS-r5.54. fasta.gz) and medfly, Ceratitis capitata (ftp://ftp.ncbi.nlm. nih.gov/genomes/Ceratitis_capitata). We installed a local database for use with Blast2Go. Using BLASTP (e-value = 1e-05), the sequences were aligned to the NCBI RefSeq invertebrate protein database (ftp://ftp.ncbi.nih.gov/refseq/ release/invertebrate/). Additional annotations were sourced from the Pfam protein domain database using InterProScan 4.8 [64]. 


\section{Comparisons of gene models}

For a comparative assessment of the overall composition of the B. tryoni gene models, we used OrthoMCL to compare the $B$. tryoni gene models to the gene models available for two other Dipterans: C. capitata and D. melanogaster. The genome of $D$. melanogaster has been thoroughly annotated and consequently has the largest set of gene models of the three species (Table 4). In contrast, the recent annotation of $C$. capitata is unpublished but the methods used to generate the underlying gene models were similar to those used in this study. Both used trained Augustus as well as SNAP and Genemark and similar multi-staged transcriptome evidence. A major difference was that our gene models were MAKER-based, while those of $C$. capitata were produced using JAMg (http://jamg.sourceforge.net). Nevertheless, the number of gene models for C. capitata was closer to that of B. tryoni (20674 versus 16710 gene models) and probably contained a similar proportion of splice variants. Nevertheless, each of the three sets of gene models are at different stages in their curation and so the present analysis was only intended to indicate the completeness if the B. tryoni genomic assembly, not the transcriptomes.

OrthoMCL compares gene models from different organisms, producing groups of protein orthlogs based on sequence similarity. OrthoMCL was run on the three sets of gene models using using default parameters, except for the Blastp all-against-all step, where a more stringent e-value of $1 \mathrm{e}-10$ was used.

\section{Comparisons with $B$. neohumeralis and $B$. jarvisi}

Substitution rates were measured by first aligning all B. neohumeralis or B. jarvisi reads to the B. tryoni assembly using the bwa-mem aligner. Homozygous SNPs for each species comparison (B. tryoni/B. neohumeralis and B. tryoni/B. jarvisi) were extracted from the Samtools mpileup file [58] using VarScan 2 [59] at sites with a minimum coverage of 10 . Custom Perl scripts were used to classify each variant position as exon, intron, 5' UTR, 3' UTR or non-coding, using the scaffoldspecific MAKER-derived gff3 files. For coding regions, synonymous versus non-synonymous substitution rates were also extracted from the MAKER-derived gff3 files using the phase information. Indels of 1 or $2 \mathrm{bp}$ were counted as non-synonomous changes.

Deletions in the $B$. neohumeralis or B. jarvisi genomes (with respect to the $B$. tryoni assembly) were identified by first mapping reads from the two other species to the $B$. tryoni assembly using bwa-mem, which allows gapped alignments. The only alignments retained were those that both consisted of paired sequencing reads and had a mapping quality greater than 20. The BEDtools utility genomeCoverageBed [55] was used to identify all B. tryoni genomic intervals over $10 \mathrm{bp}$ with zero coverage by either
B. neohumeralis or B. jarvisi Illumina HiSeq data. To further reduce any false positives due to regions of low coverage, only those deletions with $>20 x$ coverage for all the 10 bases immediately bordering both sides of the deleted segment were used in further analysis.

A gapped aligner allows even short segments of $B$. neohumeralis or $B$. jarvisi reads (19bp with a seed length of 19) to be mapped to B. tryoni even if the remainder of the read does not match. That had the advantage of maximising the coverage of the homologous sequences on either side of any B. neohumeralis or B. jarvisi deletion, which in turn reduced the likelihood of mistaking low mapping coverage with a real deletion. Conversely, this limited our ability to identify short deletions as some reads will align over short gaps and/or mismatches. However, since we were primarily interested in identifying deletions in order to develop PCR-based species identification tests, we did not quantify deletions under $10 \mathrm{bp}$.

\section{Availability of supporting data}

The data set supporting the results of this article is available in the NCBI Bioproject repository, project ID $241080 \mathrm{http}: / /$ www.ncbi.nlm.nih.gov/bioproject/?term=PRJNA241080.

\section{Additional files}

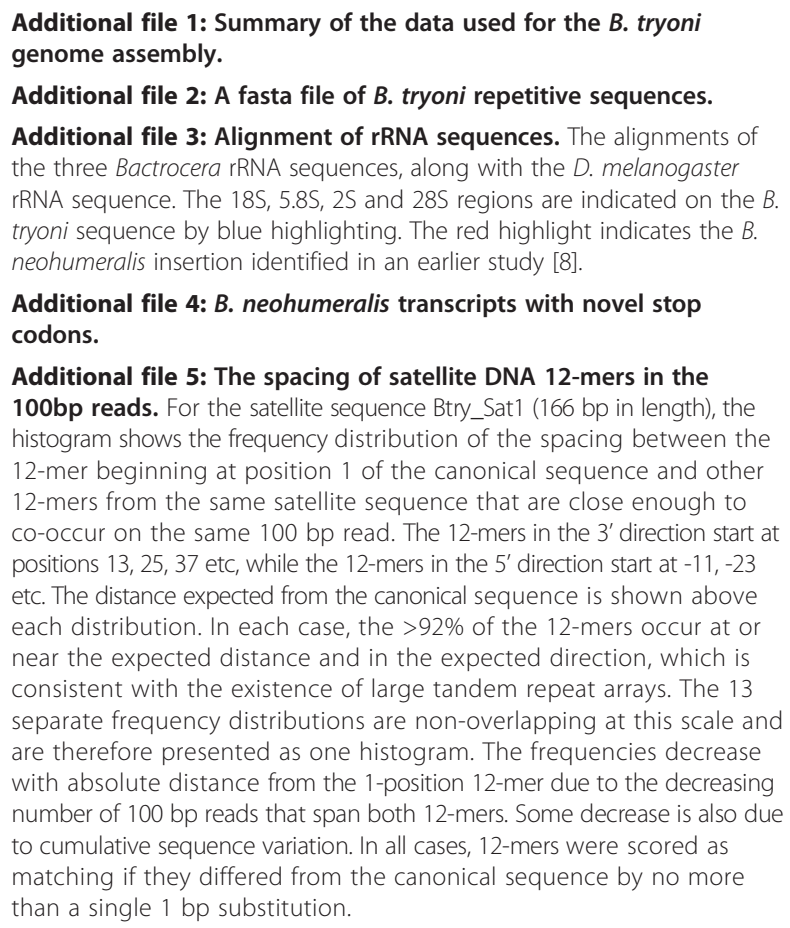

Additional file 4: $B$. neohumeralis transcripts with novel stop codons.

Additional file 5: The spacing of satellite DNA 12-mers in the 100bp reads. For the satellite sequence Btry_Sat1 (166 bp in length), the histogram shows the frequency distribution of the spacing between the 12-mer beginning at position 1 of the canonical sequence and other 12 -mers from the same satellite sequence that are close enough to co-occur on the same $100 \mathrm{bp}$ read. The 12-mers in the $3^{\prime}$ direction start at positions 13, 25, 37 etc, while the 12-mers in the $5^{\prime}$ direction start at -11, -23 etc. The distance expected from the canonical sequence is shown above each distribution. In each case, the $>92 \%$ of the 12-mers occur at or near the expected distance and in the expected direction, which is consistent with the existence of large tandem repeat arrays. The 13 separate frequency distributions are non-overlapping at this scale and are therefore presented as one histogram. The frequencies decrease with absolute distance from the 1-position 12-mer due to the decreasing number of $100 \mathrm{bp}$ reads that span both 12 -mers. Some decrease is also due to cumulative sequence variation. In all cases, 12-mers were scored as matching if they differed from the canonical sequence by no more than a single 1 bp substitution.

\section{Competing interests}

The authors declare that they have no competing interests.

\section{Authors' contributions}

ASG, DCAS, JS, MF, ND, MRW and WBS conceived the study and planned the analyses. DCAS and KR performed the molecular work. ASG carried out the 
bioinformatic and statistical analyses, with assistance from JS and ND, and discussions with WBS. ASG, DCAS, JS and MF drafted the manuscript. All authors read, commented on and approved the final manuscript.

\section{Acknowledgements}

We acknowledge funding from the Horticulture Australia and the NSW State Government Science Leveraging Fund. Work was supported by HA grant (CT10033) to ASG and DCAS, the University of New South Wales, the Victorian Department of Primary Industries and by EIF Super Science funding to the Systems Biology Initiative and the Ramaciotti Centre for Genomics.

\section{Author details}

'Evolution and Ecology Research Centre, School of Biological, Earth and Environmental Sciences, The University of New South Wales, Sydney, NSW 2052, Australia. ${ }^{2}$ Sydney Grammar School, College Street, Darlinghurst, NSW 2010, Australia. ${ }^{3}$ Systems Biology Initiative, School of Biotechnology and Biomolecular Sciences, The University of New South Wales, Sydney 2052 NSW, Australia. ${ }^{4}$ School of Biotechnology and Biomolecular Sciences, The University of New South Wales, Sydney 2052 NSW, Australia. ${ }^{5}$ Ramaciotti Centre for Gene Function Analysis, The University of New South Wales, Sydney 2052 NSW, Australia.

Received: 4 August 2014 Accepted: 12 December 2014 Published: 20 December 2014

\section{References}

1. Drew RAl: The tropical fruit flies diptera tephritidae dacinae of the australasian and oceanian regions. Memoir Queens/ Mus 1989, 26:1-521.

2. Osborne R, Meats A, Frommer M, Sved JA, Drew RAl, Robson MK: Australian distribution of 17 species of fruit flies (Diptera: Tephritidae) caught in cue lure traps in February 1994. Aust J Entomol 1997, 36:45-50.

3. White IM, Elsom-Harris MM: Fruit flies of economic significance: their identification and bionomics. Wallingford: CAB International; 1992.

4. Lewontin RC, Birch LC: Hybridization as a source of variation for adaptation to new environments. Evolution 1966, 20:315-336.

5. Tyschen PH, Fletcher BS: Studies on the rhythm of mating in the Queensland fruit fly, Dacus (Bactrocera) tryoni. J Insect Physiol 1971, 17:2139-2156.

6. Smith PH: Genetic manipulation of the circadian clock's timing of sexual behaviour in the Queensland fruit flies, Dacus tryoni and Dacus humeralis. Physiol Entomol 1979, 4:71-78.

7. Gilchrist AS, Ling AE: DNA microsatellite analysis of naturally occurring colour intermediates between Bactrocera tryoni (Froggatt) and Bactrocera neohumeralis (Hardy) (Diptera : Tephritidae). Aust J Entomol 2006, 45:157-162.

8. Morrow J, Scott L, Congdon B, Yeates D, Frommer M, Sved J: Close genetic similarity between two sympatric species of tephritid fruit fly reproductively isolated by mating time. Evolution 2000, 54(3):899-910.

9. Wang Y, Yu H, Raphael K, Gilchrist AS: Genetic delineation of sibling species of the pest fruit fly Bactocera (Diptera: Tephritidae) in Australia using microsatellites. Bull Entomol Res 2003, 93:351-360.

10. Fitt GP: Responses by female dacinae to male lures and their relationship to patterns of mating behavior and pheromone response. Entomol Exp Appl 1981, 29(1):87-97.

11. Cruickshank L, Jessup AJ, Cruickshank DJ: Intersprecific crosses of Bactrocera tryoni (Froggatt) and Bactrocera jarvisi (Tryon) in the laboratory. Aust J Entomol 2001, 40:278-280.

12. Shearman DCA, Frommer M, Morrow JL, Raphael KA, Gilchrist AS: Interspecific hybridization as a source of novel genetic markers for the sterile insect technique in Bactrocera tryoni (Diptera: Tephritidae). J Econ Entomol 2010, 103(4):1071-1079.

13. Simpson JT, Wong K, Jackman SD, Schein JE, Jones SJM, Birol I: ABySS: A parallel assembler for short read sequence data. Genome Res 2009, 19(6):1117-1123.

14. Boetzer M, Henkel CV, Jansen HJ, Butler D, Pirovano W: Scaffolding preassembled contigs using SSPACE. Bioinformatics 2011, 27(4):578-579.

15. Parra G, Bradnam K, Korf I: CEGMA: a pipeline to accurately annotate core genes in eukaryotic genornes. Bioinformatics 2007, 23(9):1061-1067.

16. Nardi F, Carapelli A, Boore JL, Roderick GK, Dallai R, Frati F: Domestication of olive fly through a multi-regional host shift to cultivated olives: Comparative dating using complete mitochondrial genomes. Mol Phylogenet Evol 2010, 57(2):678-686
17. Wang $X$, Chen W, Huang Y, Sun J, Men J, Liu H, Luo F, Guo L, Lv X, Deng C, Zhou C, Fan Y, Li X, Huang L, Hu Y, Liang C, Hu X, Xu J, Yu X: The draft genome of the carcinogenic human liver fluke Clonorchis sinensis. Genome Biol 2011, 12(10):R107.

18. Marcais G, Kingsford C: A fast, lock-free approach for efficient parallel counting of occurrences of k-mers. Bioinformatics 2011, 27(6):764-770.

19. Rizk G, Lavenier D, Chikhi R: DSK: k-mer counting with very low memory usage. Bioinformatics 2013, 29(5):652-653.

20. Zhao JT, Frommer M, Sved JA, Zacharopoulou A: Mitotic and polytene chromosome analyses in the Queensland fruit fly, Bactrocera tryoni (Diptera: Tephritidae). Genome 1998, 41(4):510-526.

21. Peterson BK, Hare EE, lyer VN, Storage $S$, Conner L, Papaj DR, Kurashima R,

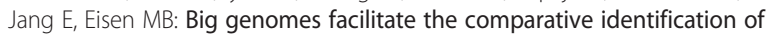
regulatory elements. PloS One 2009, 4(3).e4688

22. Tsoumani KT, Mathiopoulos KD: Genome size estimation with quantitative real-time PCR in two Tephritidae species: Ceratitis capitata and Bactrocera oleae. J Appl Entomol 2012, 136(8):626-631.

23. Hu H, Bandyopadhyay PK, Olivera BM, Yandell M: Characterization of the Conus bullatus genome and its venom-duct transcriptome. BMC Genomics 2011, 12:60.

24. Smit AFA, Hubley R: RepeatModeler Open-1.0. 2008-2010. http://www. repeatmasker.org. Accessed 20 December 2014.

25. Green CL, Frommer M: The genome of the Queensland fruit fly Bactrocera tryoni contains multiple representatives of the mariner family of transposable elements. Insect Mol Biol 2001, 10(4):371-386.

26. Bennett $\mathrm{CL}$, Frommer $\mathrm{M}$ : The white gene of the tephritid fruit fly Bactrocera tryoni is characterized by a long untranslated 5 ' leader and a $12 \mathrm{~kb}$ first intron. Insect Mol Biol 1997, 6(4):343-356.

27. Palomeque T, Lorite P: Satellite DNA in insects: a review. Heredity 2008, 100(6):564-573.

28. Macas J, Neumann P, Novak P, Jiang JM: Global sequence characterization of rice centromeric satellite based on oligomer frequency analysis in large-scale sequencing data. Bioinformatics 2010, 26(17):2101-2108.

29. Jurka J, Kapitonov W, Pavlicek A, Klonowski P, Kohany O, Walichiewicz J: Repbase update, a database of eukaryotic repetitive elements. Cytogenet Genome Res 2005, 110(1-4):462-467.

30. Smit AFA, Hubley R, Green P: RepeatMasker Open-3.0. 1996-2010. http://www. repeatmasker.org. Accessed 20 December 2014.

31. Stage DE, Eickbush TH: Sequence variation within the rRNA gene loci of 12 Drosophila species. Genome Res 2007, 17(12):1888-1897.

32. Cantarel BL, Korf I, Robb SMC, Parra G, Ross E, Moore B, Holt C, Alvarado AS, Yandell M: MAKER: An easy-to-use annotation pipeline designed for emerging model organism genomes. Genome Res 2008, 18(1):188-196.

33. Conesa A, Gotz S, Garcia-Gomez JM, Terol J, Talon M, Robles M: Blast2GO: a universal tool for annotation, visualization and analysis in functional genomics research. Bioinformatics 2005, 21(18):3674-3676.

34. Li L, Stoeckert CJ Jr, Roos DS: OrthoMCL: identification of ortholog groups for eukaryotic genomes. Genome Res 2003, 13(9):2178-2189.

35. Dunn KA, Bielawski JP, Yang Z: Substitution rates in Drosophila nuclear genes: Implications for translational selection. Genetics 2001, 157:295-305.

36. Fruit Fly (Diptera: Tephritidae) Classification \& Diversity [http://www.sel. barc.usda.gov/diptera/tephriti/TephClas.htm]

37. Malacrida AR, Gomulski LM, Bonizzoni M, Bertin S, Gasperi G, Gugliclmino CR: Globalization and fruitfly invasion and expansion: the medfly paradigm. Genetica 2007, 131(1):1-9.

38. Smith PT, Mcpheron BA, Kambhampati S: Phylogenetic analysis of mitochondrial DNA supports the monophyly of Dacini Fruit Flies (Diptera: Tephritidae). Ann Entomol Soc Am 2002, 95(7):658-664.

39. Clarke AR, Armstrong KF, Carmichael AE, Milne JR, Raghu S, Roderick GK, Yeates DK: Invasive phytophagous pests arising through a recent tropical evolutionary radiation: The Bactrocera dorsalis complex of fruit flies. Annu Rev Entomol 2005, 50:293-319.

40. Shearman DCA: The evolution of sex determination systems in dipteran insects other than Drosophila. Genetica 2002, 116(1):25-43.

41. An X, Wilkes K, Bastian Y, Morrow JL, Frommer M, Raphael K: The period gene in two species of tephritid fruit fly differentiated by mating behaviour. Insect Mol Biol 2002, 11:419-430.

42. Orr HA: Genetics of male and female sterility in hybrids of Drosophila pseudoobscura and D. persimilis. Genetics 1987, 116:555-563.

43. Kulathinal RJ, Stevison LS, Noor MA: The genomics of speciation in Drosophila: diversity, divergence and introgression estimated using low-coverage genome sequencing. PloS Genetics 2009, 5(7):e1000550. 
44. Garrigan D, Kingan SB, Geneva AJ, Andolfatto P, Clark AG, Thornton KR, Presgraves DC: Genome sequencing reveals complex speciation in the Drosophila simulans clade. Genome Res 2012, 22(8):1499-1511.

45. Meats A, Maheswaran P, Frommer M, Sved J: Towards a male-only release system for SIT with the Queensland fruit fly, Bactrocera tryoni, using a genetic sexing strain with a temperature-sensitive lethal mutation. Genetica 2002, 116(1):97-106.

46. Via S: Natural selection in action during speciation. Proc Natl Acad Sci U S A 2009, 106:9939-9946

47. Nadeau NJ, Jiggins CD: A golden age for evolutionary genetics? Genomic studies of adaptation in natural populations. Trends Genet 2010, 26(11):484.

48. Renaut $S$, Bernatchez $L \mathrm{~L}$ : Transcriptome-wide signature of hybrid breakdown associated with intrinsic reproductive isolation in lake whitefish species pairs (Coregonus spp. Salmonidae). Heredity 2011, 106(6):1003-1011.

49. Emelianov I, Hernandes-Lopez A, Torrence M, Watts N: Fusion-fission experiments in Aphidius: evolutionary split without isolation in response to environmental bimodality. Heredity 2011, 106(5):798-807.

50. Nosil P, Schluter D: The genes underlying the process of speciation. Trends Ecol Evol 2011, 26(4):160-167.

51. Streisfeld MA, Young WN, Sobel JM: Divergent selection drives genetic differentiation in an R2R3-MYB transcription factor that contributes to incipient speciation in Mimulus aurantiacus. PloS Genetics 2013, 9(3): e1003385.

52. Bolger AM, Lohse M, Usadel B: Trimmomatic: a flexible trimmer for Illumina sequence data. Bioinformatics 2014, 30(15):2114-2120.

53. Kelley DR, Schatz MC, Salzberg SL: Quake: quality-aware detection and correction of sequencing errors. Genome Biol 2010, 11(11):R116.

54. Li H: Aligning sequence reads, clone sequences and assembly contigs with BWA-MEM. eprint ArXiv 2013, 1303:3997. [http://www.arxiv.org/abs/1303.3997].

55. Quinlan AR, Hall IM: BEDTools: a flexible suite of utilities for comparing genomic features. Bioinformatics 2010, 26(6):841-842.

56. Edgar RC: MUSCLE: multiple sequence alignment with high accuracy and high throughput. Nucleic Acids Res 2004, 32(5):1792-1797.

57. Gouy M, Guindon S, Gascuel O: SeaView Version 4: A multiplatform graphical user interface for sequence alignment and phylogenetic tree building. Mol Biol Evol 2010, 27(2):221-224.

58. Li H, Handsaker B, Wysoker A, Fennell T, Ruan J, Homer N, Marth G, Abecasis G, Durbin R, Genome Project Data P: The Sequence Alignment/Map format and SAMtools. Bioinformatics 2009, 25(16):2078-2079.

59. Koboldt DC, Zhang QY, Larson DE, Shen D, McLellan MD, Lin L, Miller CA, Mardis ER, Ding L, Wilson RK: VarScan 2: Somatic mutation and copy number alteration discovery in cancer by exome sequencing. Genome Res 2012, 22(3):568-576.

60. Korf I: Gene finding in novel genomes. BMC Bioinformatics 2004, 5:59.

61. Stanke M, Tzvetkova A, Morgenstern B: AUGUSTUS at EGASP: using EST, protein and genomic alignments for improved gene prediction in the human genome. Genome Biol 2006, 7(Suppl:1:S11):11-18

62. Lukashin AV, Borodovsky M: GeneMark.hmm: new solutions for gene finding. Nucleic Acids Res 1998, 26(4):1107-1115.

63. Grabherr MG, Haas BJ, Yassour M, Levin JZ, Thompson DA, Amit I, Adiconis X, Fan L, Raychowdhury R, Zeng QD, Chen ZH, Mauceli E, Hacohen N, Gnirke A, Rhind N, di Palma F, Birren BW, Nusbaum C, Lindblad-Toh K, Friedman N, Regev A: Full-length transcriptome assembly from RNA-Seq data without a reference genome. Nat Biotechnol 2011, 29(7):644-U130.

64. Hunter S, Jones P, Mitchell A, Apweiler R, Attwood TK, Bateman A, Bernard T, Binns D, Bork P, Burge S, de Castro E, Coggill P, Corbett M, Das U, Daugherty L, Duquenne L, Finn RD, Fraser M, Gough J, Haft D, Hulo N, Kahn D, Kelly E, Letunic I, Lonsdale D, Lopez R, Madera M, Maslen J, McAnulla C, McDowall J, et al: InterPro in 2011: new developments in the family and domain prediction database. Nucleic Acids Res 2012, 40(Database issue):D306-D312.

doi:10.1186/1471-2164-15-1153

Cite this article as: Gilchrist et al:: The draft genome of the pest tephritid fruit fly Bactrocera tryoni: resources for the genomic analysis of hybridising species. BMC Genomics 2014 15:1153.

\section{Submit your next manuscript to BioMed Central and take full advantage of:}

- Convenient online submission

- Thorough peer review

- No space constraints or color figure charges

- Immediate publication on acceptance

- Inclusion in PubMed, CAS, Scopus and Google Scholar

- Research which is freely available for redistribution

Submit your manuscript at www.biomedcentral.com/submit
C Biomed Central 